\title{
ALCUNE CARATTERISTICHE DELLO SLOVENO OCCIDENTALE TRA LINGUA E PAROLA
}

\section{OGGETTO DELLA RICERCA}

Nel lontano 1992 pasammo un semestre all'Università di Udine con la borsa di studio Alpe Adria per studiare gli influssi romanzi sullo sloveno. Nell'ambito di questa ricerca effettuammo tra l'altro un'indagine riguardante gli influssi romanzi sulla lingua dei testi scritti per il concorso dialettale «Moja vas» («Il mio paese») promosso dal Centro Studi Nediža a San Pietro al Natisone (Špeter Slovenov) e riguardante gli alunni delle scuole elementari e medie della fascia di parlata slovena della provincia di Udine. ${ }^{1}$

Il presente contributo è frutto di una rilettura e di una rielaborazione linguistica di circa 100 testi dialettali, composti dall'anno 1987 fino all'anno 1991 dagli alunni tra i 7 e i 15 anni. Si tratta di temi che non sorpassano una o due pagine e in cui i bambini parlano in modo prevalente del loro paese, della loro famiglia, della scuola e della vita di tutti i giorni.

\section{PROBLEMI INCONTRATI}

\subsection{Illeggibilità, presenza delle correzioni}

L'archivio del concorso «Moja vas» conserva naturalmente gli originali dei testi, in alcuni casi (non tutti) ci si trovano anche le copie dattilografate dei temi. ${ }^{2}$ Dobbiamo ammettere che nella lettura dei primi abbiamo incontrato dei notevoli problemi di leggibilità che un testo dialettale, e per di più scritto a mano, non può non presentare a un non parlante del dialetto, tanto sul livello grafico (per la scarsa qualità delle fotocopie) quanto sul livello di comprensibilità. La comparazione delle due varianti ha mostrato però che, nella maggior parte dei casi, i testi dattilografati sono proprio quelli in cui si possono reperire certe deviazioni dall'originale, certi cambiamenti o piuttosto «correzioni» linguistiche e grammaticali. Nell'analisi dei fenomeni

\footnotetext{
* Indirizzo dell'autrice: Filozofska fakulteta, Oddelek za romanske jezike in književnosti, Aškerčeva 2, 1000 Ljubljana, Slovenia. Email: agata.sega@guest.arnes.si

${ }^{1}$ I risultati di questa indagine non sono stati ancora pubblicati, vorremmo perciò approfittare di questa occasione per ringraziare la signora Živa Gruden, direttrice del Centro scolastico bilingue a San Pietro al Natisone, ed il signor Pietro Petricig, direttore del Centro studi Nediža a Cividale, per aver messo a nostra disposizione l'archivio del concorso e per averci prestato tutto l'aiuto necessario.

${ }^{2} \mathrm{Si}$ tratta di quelli considerati i migliori e destinati ad apparire nel Vartac, la pubblicazione annuale che raccoglie testi e disegni realizzati per il nostro concorso.
} 
linguistici ci siamo dunque limitati all'uso degli originali prendendo in conto per le nostre conclusioni anche le copie dattiloscritte e preparate per la pubblicazione.

\subsection{L'(in)competenza linguistica degli autori}

Gli autori dei testi, soprattutto quelli più giovani (sappiamo già che si tratta di bambini tra i 7 ed i 15 anni), usano strutture molto semplici (soggetto - predicato complemento oggetto) e sembrano aver avuto non poche difficoltà a esprimersi per iscritto in un dialetto che, naturalmente, appartiene al parlato. Dobbiamo renderci conto che per molti di questi alunni il tema del concorso «Moja vas» è stato assolutamente il primo scritto dialettale che abbiano prodotto nella loro vita, non dobbiamo però neanche trascurare il fatto che, in ogni caso, la capacità di espressione e la competenza linguistica dei bambini è minore di quella dei parlanti dialettali adulti. Ciò vuol dire che i nostri testi sono limitati dal punto di vista non solo sintattico, ma anche lessicale: molto spesso i loro argomenti si ripetono, i bambini descrivono principalmente i loro genitori e nonni, gli animali domestici, i giocattoli e il luogo (di solito il paese, raramente la città) dove abitano, allorché le descrizioni dei lavori contadini, delle feste popolari e della vita del paese si incontrano molto più raramente. Sintatticamente e lessicalmente, questi scritti non riflettono dunque in modo completamente attendibile la vera immagine del dialetto; date le circostanze, non dobbiamo sopravvalutare o generalizzare le conclusioni a cui siamo arrivati in base a questo materiale linguistico, che, ciononostante, può offrire al linguista armato di cautela necessaria qualche informazione preziosa sulle tendenze dialettali attuali.

\section{CONCLUSIONI IN BASE AL MATERIALE LINGUISTICO ANALIZZATO}

Visto che presentano in generale le caratteristiche tipiche delle parlate slovene occidentali, la fonetica ed il lessico verranno trattati in modo relativamente superficiale. La nostra attenzione si concentrerà sulla morfosintassi, dove ci sembra di aver stabilito delle tendenze non completamente conformi a quelle descritte da Mitja Skubic nella sua monografia sugli elementi romanzi nelle parlate slovene occidentali. ${ }^{3}$

\subsection{Fonetica}

La mancanza di unità nella grafia che si nota immediatamente è comprensibile: nella maggior parte dei casi, i bambini si servono sostanzialmente della grafia italiana. Per i fonemi non esistenti nel sistema fonetico italiano ognuno cerca di trarsi d'impacccio a suo modo, per cui la grafia varia da caso a caso.

\subsection{Lessico}

L'influsso lessicale romanzo si rivela relativamente importante. Prevalgono i prestiti sostantivali (circa il 65\% dei prestiti), seguono quelli verbali che però sono già abbastanza meno numerosi (circa il 20\%). Ancora meno importante è il numero

\footnotetext{
${ }^{3}$ Skubic 2000 (traduzione italiana dell'originale sloveno Skubic 1997).
} 
degli aggettivi (circa il 10\%) e degli avverbi (circa il 4\%), mentre la percentuale delle preposizioni, interiezioni, congiunzioni o espressioni congiuntive è quasi trascurabile (l'1\%). Nella maggior parte dei casi i prestiti si adattano completamente al sistema morfologico dialettale e alle regole di flessione e si muniscono di prefissi, di suffissi oppure d'infissi sloveni (skavan «scavato», smo se ustufali «ci siamo stufati» avanzan «avvanzato», rekuperavati «recuperare», ecc.). Tra i prestiti molto recenti troviamo naturalmente più italianismi (o meglio venezianismi) che friulanismi. Ancora un'osservazione interessante: nessuno degli aggettivi imprestati accompagna il verbo, tutti adempiono la funzione di complemento predicativo.

\subsection{Morfosintassi}

Nel campo delle tendenze morfosintattiche causate o solo favorite dall'influsso romanzo si può parlare di due livelli, cioè delle tendenze morfosintattiche generali da una parte e di quelle individuali dall'altra.

3.3.1. Tendenze morfosintattiche generali: $\mathrm{Si}$ tratta di tendenze comuni a tutti o quasi tutti i testi esaminati, perfino a quelli (molto pochi) scritti in sloveno standard, ${ }^{4}$ che però non possono nascondere mai l'origine occidentale dell'autore. Parliamo dunque di tendenze generali, appartenenti al concetto saussuriano di lingua perché caratteristiche, secondo tutte le apparenze, anche del resto dei parlanti dello sloveno occidentale. Queste tendenze sono state sistematicamente presentate e commentate nella sopra menzionata monografia (Skubic 2000).

Nello studio degli influssi romanzi bisogna però fare una distinzione molto netta tra i fenomeni limitati alle parlate slovene occidentali e quelli che caratterizzano anche lo sloveno parlato (cioè la lingua non colta, «lo sloveno neostandard») oppure i dialetti sloveni delle altre regioni. Un esempio: nei nostri testi si incontrano il pronome dimostrativo ta «questo» nella ruolo di «articolo definito» ed il numerale en, ena, eno nel ruolo di «articolo indefinito», una caratteristica che potrebbe venire considerata come conseguenza dell'influsso romanzo. Traducendo però le stesse frasi nello sloveno parlato (nel nostro caso nella parlata di Lubiana, ma sarebbe uguale anche altrove) possiamo constatare che gli «articoli» rimangono ai loro posti:

Te parve hiše so ble narete uos les an uos iluco $(202 / 88, \mathrm{~F} 15)^{5}$ /ta prve hiše/ «le prime case erano fatte di legno e di argilla»;

/.../ an miesac od tega je paršla ta tracia Giulia (323/87, F12) / ta tretja Julija/ «un mese fà è arrivata la terza Giulia»;

/.../ se darši ku na balerina (323/87, F12) /ena balerina/ «ha assunto l'aria da ballerina».

\footnotetext{
${ }^{4}$ In disaccordo con il regolamento del concorso che richiede il dialetto.

${ }^{5} 202$ = il numero del testo nell'archivio; $88=1988$, cioè l'anno in cui il testo è stato scritto; $\mathrm{F}(\mathrm{o} \mathrm{M})=$ sesso dell'autore; 12 = età dell'autore.
} 
Il cosiddetto «articolo» appare - e secondo noi questo prova appunto quello che stiamo dicendo - anche nei pochi temi che sono (o si vogliono) scritti in sloveno standard:

Jaz imam enega psička $(2 / 88, \check{Z} 7)$ «io ho un cagnolino».

I casi in cui si potrebbe parlare con certezza dell'uso dell'articolo sotto l'influsso romanzo sono rarissimi. Nel caso

/.../ kar bon miela ano dialo (202/88, Ž15) «quando avrò un lavoro».

bisogna osservare però che si tratta di un calco linguistico, perché si vede subito che l'intera struttura è tradotta direttamente dall'italiano. ${ }^{6}$ La frase seguente potrebbe rappresentare un altro esempio possibile dell'influsso romanzo, perché in questo caso lo sloveno parlato o dialettale non si servirebbe dell «articolo»:

/.../ nose nimar an facul čarin $(198 / 88$, F11) /zmeri nos črno ruto/ «porta sempre un foulard nero».

Bisogna sottolineare che, non di rado, una tendenza già esistente nella lingua si mantiene e perfino si rinforza proprio grazie all'influsso dell'adstrato. Dobbiamo comunque osservare che la presenza dell'influsso romanzo non è sempre facilmente provabile. Ci limiteremo solo a due tendenze morfosintattiche, cioè l'indebolimento del neutro e l'uso del nominativo come unica forma flessiva, quindi proprio quelle due per cui il nostro materiale rivela una situazione linguistica differente da quella stabilita dallo Skubic dopo l'esame di una ricca base di fonti dialettali (Skubic 2000: 60-62).

3.3.1.1. L'indebolimento del neutro: L'indebolimento del neutro è un fenomeno conosciuto nella dialettologia slovena ${ }^{7}$ che non può essere attribuito - almeno non esclusivamente - all'influsso romanzo o straniero in generale. In quanto a questo problema, i risultati delle ricerche di Mitja Skubic differiscono dai nostri e da quelli di Spinozzi Monai (1995a e 1995b), che prende in considerazione anche i risultati delle inchieste dirette su campo. ${ }^{8}$ Constatando l'indebolimento sporadico del neutro nelle sue fonti ed accennando alla presenza dello stesso fenomeno nei dialetti sloveni centrali, lo Skubic afferma che «la categoria del neutro appare ben salda tanto nella tradizione orale quanto, ad esempio, negli scritti degli scolari»» (Skubic 2000: 69; sottolineato da A.S.). I nostri testi mostrano però uno stato delle cose completamente diverso; abbiamo notato infatti che presentano difficoltà

\footnotetext{
${ }^{6}$ In sloveno standard si direbbe »ko bom imela službo, ko bom v službi, bom zaposlena«.

${ }^{7}$ Cfr. per esempio Priestly 1984. L'indebolimento del neutro non viene menzionato da Fran Ramovš tra le caratteristiche dei dialetti sloveni sudorientali (Ramovš 1935: 42-105).

${ }^{8}$ Si basa tra l'altro sulle risposte di 9 informatori a Sorzento/Sarženta nel Commune di San Pietro al Natisone/Špeter e Montemaggiore/Matajur e Masseris/Mašere nel Comune di Savogna/Sovodnje.
} 
proprio tutti i sostantivi del genere neutro senza eccezione. Nell'uso dei sostantivi di genere neutro, i bambini riscontrano notevoli problemi: sembrano infatti non essere in grado di decidere quale genere verrebbe attribuito a questi sostantivi. Così succede che lo stesso sostantivo, usato più di una volta nello stesso testo, venga trattato ora come femminile, ora come maschile. ${ }^{9}$ Nel testo $320 / 87$ per esempio, un ragazzo di 13 anni scrive dapprima «naše praseta opite su ble» («i nostri porci erano ubriachi») e solo poche linee più avanti «praseta so pjani» («i porci sono ubriachi»). Vediamo un altro esempio estremamente istruttivo nel quale il sostantivo korito «trogolo, vasca per la raccolta dell'acqua», neutro in sloveno standard, cambia genere non meno di sette volte, ma si presenta sempre come maschile o come femminile, mai come neutro:

Seučanski so misnli kostruit an korito ki sada so dvia. Pa an krat je biu an an treti. Te parvi korito je biu naret na leta 1861 al 1871 /.../. Je bla usa skavana tu an velik kaman /.../. Sada tist korito ga nia vic an ist ga niasam mai vidla. /.../ Je muoru bit pru liep! Te drugi so ga nardil kar je bila uoiska od '15/'18. So jo nardil vojaki ki so se usafali tle. Tele korito je šele tu vas an stoj na dolenie Seuce. Te treci so jo nardil na leta 1947 an je te narbui nuov. /.../ An krat tu hiše niso mial vode, an korite so šle pru dobro (202/88, F15).

A volte, la scelta del genere rivela e conferma concretamente l'influsso del modello romanzo, come per esempio il genere maschile seguente:

An danas, ki so pasali puno lieta (202/88, F15) «sono passati tanti anni».

3.3.1.2. L'uso del nominativo come unica forma flessiva: L'indebolimento della flessione si fa vedere il più delle volte nell'uso del caso sbagliato dopo una preposizione. Nelle sue fonti, Mitja Skubic nota esempi in cui «la preposizione scelta e la forma flessionale del sostantivo non concordano», sottolineando però che «tali discrepanze appaiono nei giornali odierni, mentre sono quasi inesistenti nel materiale folclorico, sarebbe a dire, nelle forme spontanee della lingua parlata» (Skubic 2000: 76)..$^{10}$

Ecco qualche esempio tratto dal nostro materiale dialettale:

/.../ z bičikleto brez kolami mali (323/87, F12); ${ }^{11}$

/.../ an cos meso $(345 / 87, \mathrm{~F} 13) ;^{12}$

Te parve hiše so ble narete uos les an uos iluco $(202 / 88$, F15).

\footnotetext{
${ }^{9}$ Fenomeno notato anche da Liliana Spinozzi Monai (1995b: 421).

${ }^{10}$ Questa tendenza non manca di rivelarsi anche nella lingua dei mezzi di comunicazione sloveni. Stiamo parlando soprattutto dell'uso della forma dativa del sostantivo maschile accanto ad una preposizione che richiederebbe il locativo.

${ }^{11}$ La struttura dimostra che la bambina riscontra notevoli problemi con il caso che regge la preposizione brez, poiché la usa con due casi differenti (kolami - locativo, mali - nominativo) dei quali nessuno è quello giusto, cioè il genitivo.

${ }^{12} \mathrm{Si}$ vede dalla fotocopia che la ragazza ha scritto mesa in un primo momento, ma si è corretta più tardi.
} 
Il numero elevato di casi simili, nei testi che abbiamo analizzato, dimostra secondo la nostra opinione che la tendenza verso l'indebolimento della flessione del sostantivo si è rinforzata ed estesa in modo notevole. In questo processo, l'influsso romanzo ha giocato senza dubbio un ruolo importante. Un segno ancora più evidente dell'indebolimento della declinazione è l'uso del nominativo come unica forma flessiva. Parlando di questo fenomeno, Mitja Skubic deduce dal suo materiale linguistico che «...estremamente rari sono gli esempi dove si potrebbe intravvedere la perdita della flessione del sostantivo, vale a dire, l'impiego di una sola forma flessiva» (Skubic 2000: 79). I nostri testi non corroborano questa conclusione, al contrario: tale uso appare in un gran numero di testi, in certi di loro persino più volte, manifestandosi addirittura nelle produzioni scritte dai ragazzi più grandi. Ecco qualche esempio:

/.../ zakliučni prasnik od vrtec in slovenska šola (171/87, F14);

/.../ do cilj (171/87, F14);

/.../ mi od pošolski pouk (171/87, F14);

/.../ usi otroci od vartac (323/87, F12);

/.../ je imela an star malin od vas $(202 / 88, \mathrm{~F} 15)$;

/.../ malin je biu blisu potok Rjeka (202/88, F15).

Rischiamo di incontrare un nominativo al posto di un caso obliquo persino accanto ai verbi transitivi diretti:

/.../ za narest na maihana gara od risbe (171/87, F14).

Dobbiamo osservare però che in questo tipo di casi l'oscillazione tra il nominativo ed il caso obliquo è spesso ancora sensibile:

/.../ usi so letal za usafat no jagodo, an velik kaman, no živo mraulico, na marietica, an použ, an kostan list, no vejo, en korenina, an regat an ena zlatica (171/87, F14).

\subsubsection{La spiegazione possibile delle discrepanze nei risultati nelle due ricerche:}

Abbiamo visto che i risultati delle nostre indagini non concordano completamente con quelli presentati da Mitja Skubic nel suo libro sugli elementi linguistici romanzi nello sloveno occidentale. Quali potrebbero essere le ragioni di queste discrepanze? La prima si nasconde senza dubbio nel problema sopra menzionato della mancanza di competenza linguistica dialettale dei nostri autori ed è dunque legata alla stessa scelta dei testi analizzati. La seconda ragione si rivela solo dopo la comparazione dei testi originali con le copie dattiloscritte esistenti: i materiali linguistici pubblicati nella pubblicazione Vartac, usata come fonte dialettale dallo Skubic, non riflettono in modo completamente fedele la situazione linguistica tra $i$ partecipanti al concorso. Non solo che i testi preparati per la pubblicazione appartengono agli autori linguisticamente più competenti, il che è comprensibile, 
ma si può vedere che sono stati anche corretti prima di essere pubblicati. Le correzioni apportate sono minime e molto discrete, ma riguardano proprio i fenomeni linguistici considerati come «i più gravi errori» che potrebbero infatti persino disturbare la comprensione del testo. L'uso del nominativo come unica forma flessiva ed il cambio ripetuto di genere dello stesso sostantivo ne fanno sicuramente parte.

3.3.2. Tendenze morfosintattiche individuali: Parleremo adesso delle tendenze confermate solo in alcuni testi; in altri termini, ci manterremo al livello saussuriano della parola. Le caratteristiche che lasciano intravvedere gli inizi di certe tendenze in via di farsi valere e di generalizzarsi nel dialetto, sono reperibili normalmente solo nei testi che testimoniano di un livello relativamente alto d'inquinamento linguistico. Dobbiamo distinguerle però in primo luogo di quelle che marcano un certo parlante o scrivente (e alle quali possiamo dunque attribuire il termine di caratteristiche individuali) e, in secondo luogo, da quelle che appaiono una sola volta presso un individuo. Stiamo parlando dei lapsus calami e dei diversi influssi momentanei provenienti spesso dal contesto. Chi potrebbe giudicare con certezza se nell'esempio

/.../ an list kier gor je blua napisano kier je biu zaklad (171/87, F14)

si tratta della coincidenza degli avverbi sloveni kjer e kje sotto l'influsso romanzo o di un semplice errore individuale della ragazza che, sotto l'influsso del primo kier, non ha fatto che ripetere la stessa forma aggiungendo la $r$ finale senza rendersene conto. Il problema non è affatto semplice: attira l'attenzione la presenza di alcuni casi simili, menzionati dallo Skubic (Skubic 1997: 82), ${ }^{13}$ nelle omelie dell'Attems, con la sola differenza che lì si trova la forma locativa interrogativa al posto di quella relativa ${ }^{14}$ mentre nel nostro caso si ha a che fare con la situazione inversa. Siccome egli non ha rintracciato casi simili in nessun altro testo di tutti quelli che aveva analizzato, questo caso non è stato preso in considerazione dallo Skubic perché non si devono fare conclusioni generali sull'uso individuale. Si tratta dunque dello stesso tipo di difficoltà con il quale ci incontriamo anche noi, vale a dire con il problema della distinzione tra i fenomeni della lingua e quelli della parola: a causa del possibile influsso del contesto non siamo in grado di provare l'esistenza dell'eventuale influsso romanzo nel nostro caso, che potrebbe mettere in una luce molto differente anche l'esempio citato dallo Skubic.

\footnotetext{
${ }^{13}$ Il paragrafo a cui ci riferiamo non appare nella traduzione italiana.

${ }^{14}$ Non si tratta di avverbi, ma di pronomi interrogativo e relativo: l'Attems usa $g d u$, cioè $k d o$ in sloveno letterario, al posto di kdor. Cfr. Skubic (1997: 82) che cita l'edizione a cura di Lojzka Bratuž del 1993 (Karel Mihael Attems, Slovenske pridige. Trieste: Založništvo tržaškega tiska).
} 


\section{Bibliografija}

Priestly, Tom M.S. (1984) »O popolni izgubi srednjega spola v selščini: raznodobna rekonstrukcija.» Slavistična revija 32/4, 357-372.

Ramovš, Fran (1935), Historična gramatika slovenskega jezika. VII. Dialekti. Ljubljana: Učiteljska tiskarna.

SkUBIC, Mitja (1997) Romanske jezikovne prvine na zahodni slovenski jezikovni meji. Ljubljana: Znanstveni inštitut Filozofske fakultete.

SkubIC, Mitja (2000) Elementi linguistici romanzi nello sloveno occidentale. Roma: Il Calamo.

SPINOzZI MonAI, Liliana (1995a) »La categoria del genere in un'area di contatto slavo-romanza.« Ce fastu? 71/2, 171-189.

SPINOZZI MoNAI, Liliana (1995b) »Kategorija spola v nadiškem narečju: nekaj vprašanj.« Slavistična revija 43/4, 411-425.

\section{Povzetek}

\section{NEKAJ ZNAČILNOSTI ZAHODNE SLOVENŠČINE MED JEZIKOM in GOVOROM}

$\mathrm{V}$ prispevku avtorica primerja rezultate jezikovno-interferenčne analize omejenega fonda približno 100 otroških narečnih besedil, nastalih v okviru natečaja Moja vas, ki ga vsako leto prireja Študijski center Nediža v Beneški Sloveniji, z rezultati širše zastavljenih jezikoslovnih raziskav, ki so predstavljeni v monografiji Mitje Skubica Romanske jezikovne prvine na zahodni slovenski jezikovni meji. Komentira razlike, ki se pojavljajo zlasti na področju morfosintakse, in skuša poiskati razloge zanje, ob tem pa s primeri ponazarja in opozarja, kako pomembno je pri tovrstnih raziskavah zavedanje o razlikovanju med jezikom in govorom. 